\title{
Vascular events may predict the prognosis of patients with chronic myeloid leukemia
}

\author{
Hiroshi Ureshino ${ }^{1,2}$ (1) $\cdot$ Kazuharu Kamachi ${ }^{1,2}$
}

Received: 19 March 2020 / Accepted: 22 May 2020 / Published online: 12 June 2020

(c) Japanese Society of Hematology 2020

To the Editor

Mori et al. reported the pharmacokinetics of dasatinib in a hemodialysis patient with chronic myeloid leukemia (CML), and we read with great interest the paper [1]. The patient developed severe vascular toxicities due to second generation tyrosine kinase inhibitors (TKIs) (hypertensive nephrosclerosis due to nilotinib, and heart failure due to dasatinib), but achieved a favorable treatment response with $\mathrm{MR}^{4.5}$ and maintained treatment-free remission (TFR). The phosphoproteomic signature in endothelial cells, which can regulate leukemic stem cells in CML [2], reflects the vascular toxicities of TKIs [3]. Considering the treatment response and vascular toxicity in the patient, the impairment of CML leukemic stem cell harboring from endothelial cells by TKIs may be involved in the treatment response or maintenance of TFR via endothelial dysfunction by TKIs. Although patients with severe renal insufficiency like the present patient are generally excluded from clinical trial settings, real world cohort data to investigate treatment responses or TFRs are necessary to clarify the association between vascular adverse events and treatment response or achievement of TFR in patients with CML.
Author contributions $\mathrm{HU}$ and KK wrote the manuscript. All authors approved the final version.

Funding This work was supported by research grants from JSPS KAKENHI (19K17860, HU).

\section{Compliance with ethical standards}

Conflict of interest The authors declare no conflicts of interest.

\section{References}

1. Mori J, Oshima K, Tanimoto T, Ishizuka H, Kimura S, Miura M. Pharmacokinetics of dasatinib in a hemodialysis patient with chronic myeloid leukemia and chronic kidney disease. Int J Hematol. 2020. https://doi.org/10.1007/s12185-020-02846-5.

2. Zhang B, Nguyen LXT, Li L, Zhao D, Kumar B, Wu H, et al. Bone marrow niche trafficking of miR-126 controls the self-renewal of leukemia stem cells in chronic myelogenous leukemia. Nat Med. 2018;24:450-62.

3. Gopal S, Lu Q, Man JJ, Baur W, Rao SP, Litichevskiy L, et al. A phosphoproteomic signature in endothelial cells predicts vascular toxicity of tyrosine kinase inhibitors used in CML. Blood Adv. 2018;2:1680-4.

Publisher's Note Springer Nature remains neutral with regard to jurisdictional claims in published maps and institutional affiliations.

Hiroshi Ureshino

sr0795@cc-saga-u.ac.jp

1 Division of Hematology, Respiratory Medicine and Oncology, Department of Internal Medicine, Saga University, 5-1-1 Nabeshima, Saga 849-8501, Japan

2 Department of Drug Discovery and Biomedical Sciences, Saga University, Saga, Japan 